\title{
ON- and OFF-Pathways Form Separate Neural Substrates for Motion Perception: Psychophysical Evidence
}

\author{
Christian Wehrhahn and Dietmar Rapf \\ Max-Planck-Institut für biologische Kybernetik, D-7400 Tübingen, Germany
}

\begin{abstract}
We have tested the hypothesis that in humans the signals carried by $\mathrm{ON}$ - and OFF-pathway respectively are processed for the perception of motion by two distinct physiological substrates. In vertebrates, onset of a bright visual stimulus is signaled to the CNS by ON-center retinal ganglion cells; onset of a dark stimulus is transmitted by OFF-center cells. We chose apparent motion generated by successive presentation of two adjacent lines as a stimulus. Lines presented on a bright background were either darker or brighter than this background. Delayed onset of a pair of bright or dark lines elicits apparent motion at the same time fullfilling the constraint of stimulating either ON- or OFF-center ganglion cells, respectively. We determined the threshold delay needed for subjects to perceive the temporal order of the onset of the two lines for various angular separations.

The threshold delay for a pair of bright lines stayed low for separations from 2' to $7^{\prime}$. The threshold delay for a pair of dark lines was low only within a narrow range of separations centered around $3^{\prime}$. The variation of thresholds with line distance must reflect the existence of a limited processing zone for the perception of motion. The diameter of the processing zone for bright lines is about twice as large as that for dark lines. This suggests that in humans the separation of ON- and OFF-pathways extends to the early stages of motion perception.
\end{abstract}

To test this hypothesis independently, thresholds were determined when a bright and a dark line were presented in succession. This was done for a separation of $3^{\prime}$ where thresholds for a pair of lines with equal contrast are similarly low. Temporal order was perceived correctly only when the delay was at least two to four times as high as the threshold delays found for the equal contrast stimuli.

The task of relating visual perception to central nervous function must begin by considering the functional and anatomical properties of the early stages in the visual system (Schiller and Logothetis, 1990; Shapley, 1990). In primates, onset of a bright visual stimulus is signaled to the CNS by ON-center retinal ganglion cells, while onset of a dark stimulus is transmitted by

Received Sept. 25, 1991; revised Dec. 26, 1991; accepted Jan. 13, 1992

T. Kumar provided the interface connecting computer and monitor. This work was supported by the Deutsche Forschungsgemeinschaft (SFB 307, Teilprojekt A5). T. Wiegand prepared the figures. T. S. Collett, K. Kirschfeld, T. Wachtler, and $H$. Wässle read and criticized earlier versions of the manuscript. We thank them all.

Correspondence should be addressed to Christian Wehrhahn, Max-Planck-Institut für biologische Kybernetik, Spemannstrasse 38, D-7400 Tübingen, Germany.

Copyright $\odot 1992$ Society for Neuroscience $0270-6474 / 92 / 122247-04 \$ 05.00 / 0$
OFF-center cells (Wiesel and Hubel, 1966; Famiglietti and Kolb, 1976; Bolz et al., 1982; Kremers et al., 1991). The anatomical separation of ON- and OFF-pathway seems complete in retina and LGN and is in part maintained in the primary visual cortex (Hubel and Wiesel, 1968; Schiller, 1982; Hubel and Livingstone, 1990).

Exner (1875) analyzed the discrimination of the temporal order of two successively flashed adjacent sparks and found that the temporal order was identified correctly with angular separations as small as $2^{\prime}$ and asynchronics of $15 \mathrm{mscc}$. Hc concluded from these and other experiments that motion perception in man is a sensory quality in its own right and is not derived from successive measurement of position. More recently, apparent motion and real motion were shown to be very similar with respect to the perceived velocity range (McKee, 1981). It was also suggested that on a local level, both apparent motion and real motion are processed by the same neural substrate (Biederman-Thorson et al., 1971). In this study, we chose apparent motion generated by successive onset of two lines as a stimulus. When the lines are presented on a bright background, they may be either brighter or darker than this background. Delayed onset of a pair of bright or dark lines elicits apparent motion at the same time fullfilling the constraint of stimulating either ON- or OFF-center ganglion cells, respectively. We determined the threshold delay needed for subjects to perceive the temporal order of the onset of the two lines for various angular scparations. On the basis of the evidence found, we propose that the separation of ON- and OFF-pathways extends to the early stage of visual motion perception.

Since the effect of our stimuli on the retinal ganglion cells of the subjects is of vital importance for the interpretation of our results, a short description of these effects as inferred from physiological experiments in monkeys is given here. The response of phasic ON-center retinal ganglion cells in the monkey to an increment in intensity as applied in our experimental conditions decays to the level of spontaneous activity about $50 \mathrm{msec}$ after stimulus onset. A similar time is found for OFF-center cells in response to a decrement in intensity (Kremers et al., 1991). It is most likely that these cells carry the signals processed in the perception of motion (reviewed by Schiller and Logothetis, 1990). Although tonic ganglion cells in monkeys are predominantly activated by stimuli containing chromatic contrasts, they also respond to achromatic luminance steps. This response decays to the level of spontaneous activity after about $1000 \mathrm{msec}$ (B. B. Lee, personal communication). Onset of a bright line on top of the bright background leads to an increase in the discharge rate of $\mathrm{ON}$-center ganglion cells and to a small suppression of spontaneous activity in OFF-center cells, whereas onset of a dark line will cause an increase in the activity of OFF-center cells and a small suppression of the spontaneous activity of $\mathrm{ON}$ - 
a

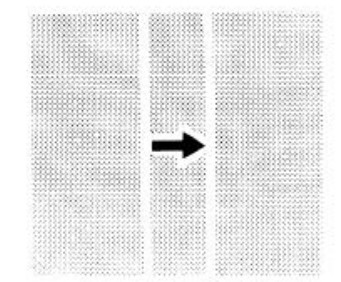

12

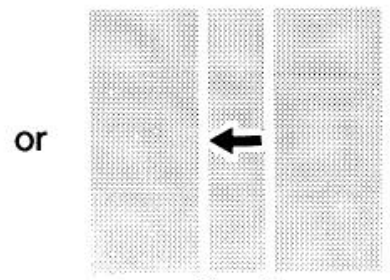

21

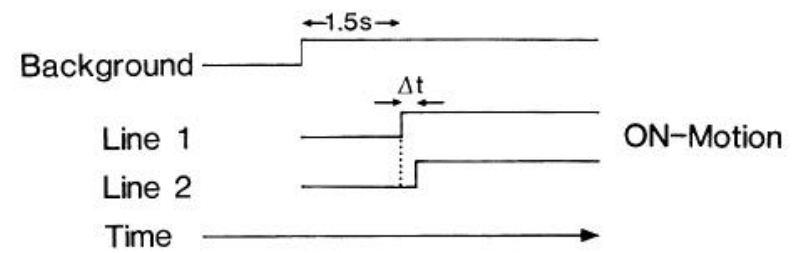

b
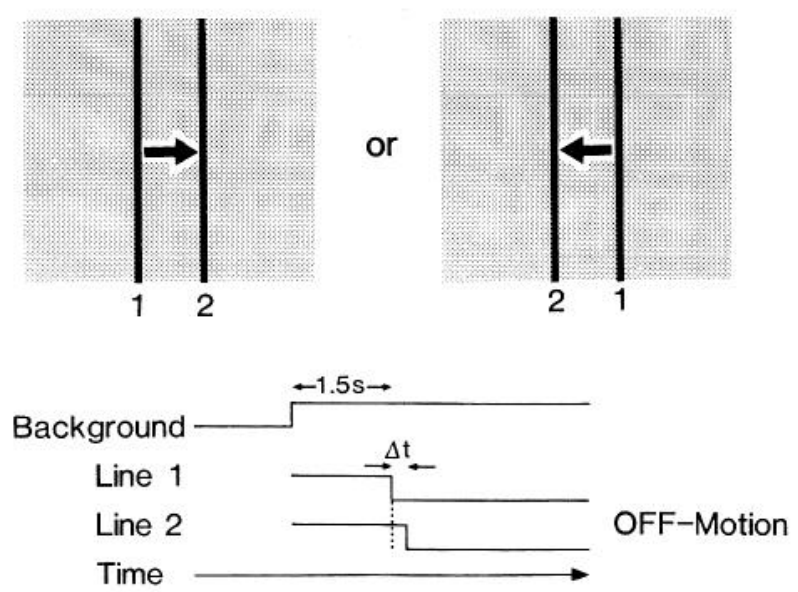

Figure 1. Sketch of the stimuli used in the experiments. A bright rectangle (22' wide and $20^{\prime}$ high) with a luminance of $82 \mathrm{~cd} / \mathrm{m}^{2}$ was switched on $1.5 \mathrm{sec}$ prior to the test stimulus. Two lines whose angular separation could be varied were presented successively on this background. Both lines were either brighter $(a)$ or darker $(b)$ than the surrounding rectangle. Note that switching on of a bright line is equivalent to increasing the local illumination whereas switching on of a dark line is equivalent to decreasing local illumination. The timing diagrams sketch the changes of illumination for the two stimulus conditions. The parameter of the experiment was the delay between the onset of the two lines $(\Delta t)$. The temporal order was random and had to be determined by the subject. All stimuli were presented to the fovea. The subjects had normal or corrected to normal vision. Stimuli were viewed binocularly using a head rest. For other details, see text.

center cells. We make the specific assumption that only increments in firing rate transmit visual information in the CNS, since the sustained firing of visual neurons decreases up to the cortical level.

\section{Materials and Methods}

Stimuli were generated on the screen of a monitor (Hewlett Packard model 1345A) equipped with a fast phosphor (P4) whose decay time to $1 \%$ of the maximal intensity is less than $0.3 \mathrm{msec}$. The signals to the monitor were controlled through an interface by a computer (IBM-PC/ AT-clone). The distance between the observer and the monitor was 3 $\mathrm{m}$. All stimuli were presented to the fovea and viewed binocularly using a head rest. The room was dimly lit by a tungsten lamp. Fixation of the subjects was guided by four red LEDs outlining a rectangle of $1.2^{\circ} \times$ $1.2^{\circ}$.
In all experiments, a bright rectangle $20^{\prime}$ high and $22^{\prime}$ wide witli a fixed luminance of $82 \mathrm{~cd} / \mathrm{m}^{2}$ appeared on the screen $1.5 \mathrm{sec}$ prior to the stimulus. (In control experiments, the background was presented 3.5 sec prior to the stimulus; this did not alter the results). The variable in the experiments was the onset asynchrony of two adjacent lines, each $0.4^{\prime}$ wide and $20^{\prime}$ high, that were either brighter or darker than the surrounding rectangle (Fig. 1). We will refer to motion induced by delayed onset of bright lines as ON-motion (i.e., motion of an object brighter than its surround) and to motion induced by delayed onset of dark lines as OFF-motion (i.e., motion of an object darker than its surround). Contrast of lines was defined as $m=\left(L_{1}-L_{0}\right) /\left(L_{1}+L_{0}\right)$, where $L_{1}$ is the luminance of a line, and $L_{0}$, that of the surrounding bright rectangle (Michelson, 1927). $m$ is positive when the line is brighter than the background and negative when darker. Contrast for bright lines was 0.58 , and for dark lines, -0.56 . The temporal order of the onset of the lines was varied at random. In each presentation either the right or the left stimulus led by $2, n, 2 n, 3 n$, or $4 n$ msec at random. $n$ could be set separately for each session (Fig. 1). Timing was controlled by a quartz clock circuit and checked by a high-precision oscilloscope with the help of a photomultiplier. The angular separation of the lines was selected from a series of values ranging from $1^{\prime}$ to $10^{\prime}$.

The observer had to indicate the temporal order of the appearance of the lines by deciding which of the two stimuli came last (which is equivalent to the direction of apparent motion) and press either the left or the right button of a "mouse" connected to the computer. All observers gave 440 such responses for each tested combination of stimulus contrast and angular separation. Data were accumulated in four sessions distributed over several days. Error feedback was provided, but control experiments indicated that this was not essential for the phenomena described. Probit curve fitting of the resulting psychometric curve gave a threshold value (and its $95 \%$ confidence interval) for that asynchrony at which the observer could correctly identify the direction of the presented sequence on $75 \%$ of the occasions. The authors and two associates, all with normal or corrected-to-normal vision, served as subjects in the experiments.

\section{Results and Discussion}

In Figure 2 the thresholds for ON-motion (open circles) and OFF-motion (solid circles) are plotted as a function of the angular separation of the two lines. In the four subjects tested, thresholds for ON-motion stay low from $2^{\prime}$ up to $7^{\prime}$ angular separation, much as in earlier experiments in which apparent motion was induced by flashing two adjacent bright lines on a dark ground (Westheimer and McKee, 1977; Westheimer, 1983). This indicates that apparent motion elicited by flashed bright lines and $\mathrm{ON}$-motion is processed by the same neuronal structures and that the ON-pathway dominates in stimulus conditions applied in the earlier experiments.

In contrast, thresholds for OFF-motion are low only within a narrow range of separations centered around $3^{\prime}$. The width of the minimum at half height is less than $3^{\prime}$. For angular separations larger than 3-4', thresholds for OFF-motion are significantly higher than those for ON-motion. Such a response could be achieved by a movement detector with two inputs separated by $3^{\prime}$ or roughly six cones in the central fovea. The perception of OFF-motion depends more strongly on line separation as compared to the perception of ON-motion.

At a separation of $3^{\prime}$, thresholds for ON- and OFF-motion are similarly low. This indicates that the OFF-pathway is able to transmit small time differences as well as the ON-pathway. The sharp increase of thresholds at $5^{\prime}$ separation found for a pair of dark lines and the clear difference to thresholds found for a pair of bright lines for distances up to 7 ' requires an explanation. The diameters of the central parts of the receptive fields of ON- and OFF-center retinal ganglion cells in the monkey seem to be similar (Rodieck et al., 1985). Thus, the different shape of the distance functions must reflect either (1) the operation of two physiologically distinct systems for the perception 

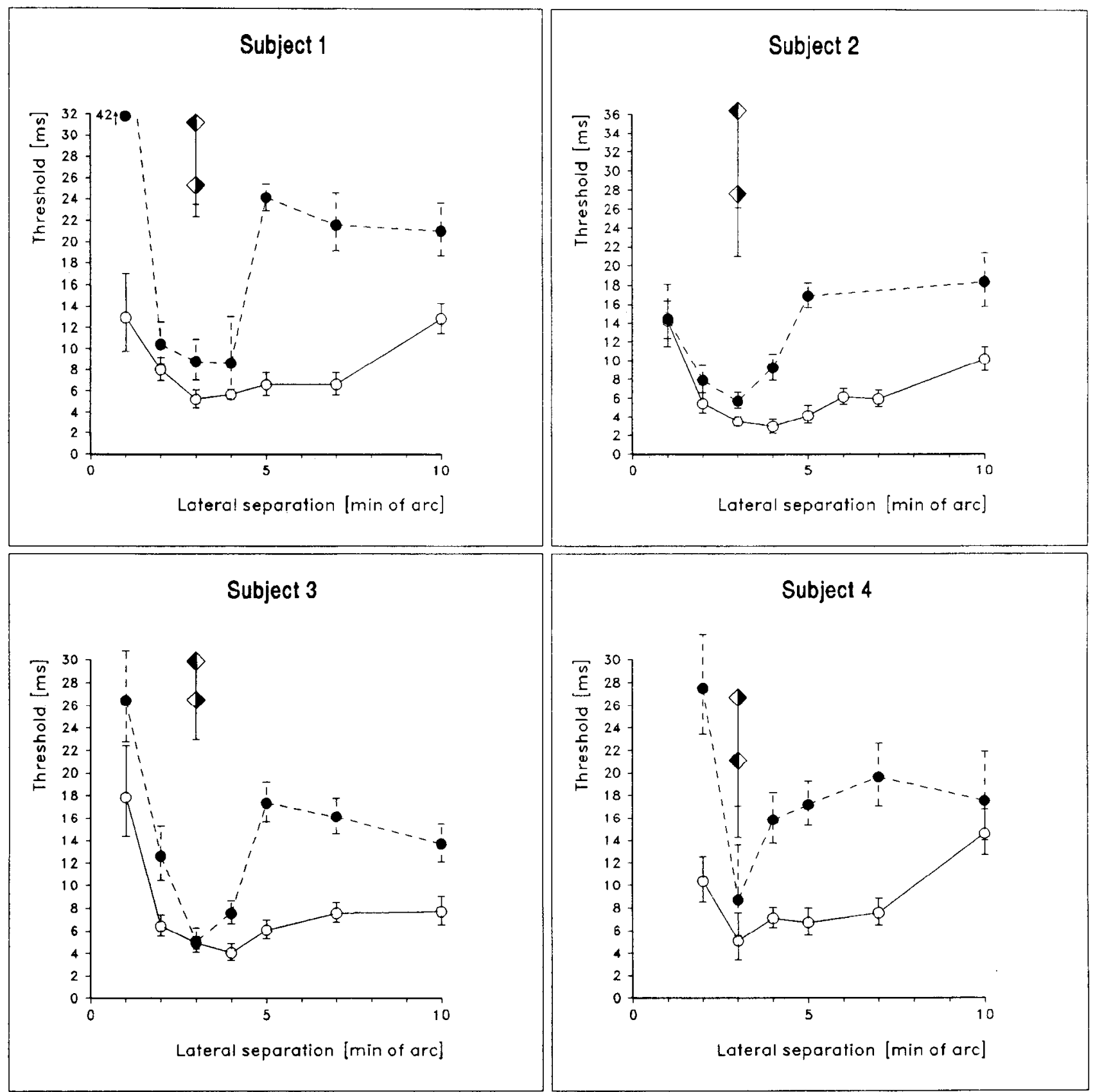

Figure 2. Threshold delays needed to perceive the temporal order of the onset of two adjacent lines as depicted in Figure 1. Thresholds are plotted as a function of the angular separation for bright lines (open circles) and dark lines (solid circles). In all subjects tested, thresholds measured for a pair of bright lines stay low, between $2^{\prime}$ and 7 ' angular separation. In contrast, thresholds found for a pair of dark lines are low only within a narrow range of separations centered around 3', staying high otherwise. Diamonds represent thresholds for successive presentation of a bright and a dark line (right half of diamond black) and vice versa (left half of diamond black) at an angular separation of $3^{\prime}$. Apparent motion induced by two lines of opposite contrast can only be seen after delays that are two to four times as long as those required for a pair of lines with equal contrast, either bright or dark. Stimuli were viewed with the central fovea (4 subjects, 440 presentations per point; error bars give $95 \%$ confidence interval for the thresholds). For opposite contrast stimuli, only the lower part of the $95 \%$ confidence interval is drawn. For other details, see Figure 1 caption.

of local motion, one presumably driven by ON-center and the other by OFF-center retinal ganglion cells, or (2) different access of the two pathways onto the cellular elements generating motion signals.

Earlier experiments designed to test the separation of ONand OFF-pathways in the perception of motion revealed equal performance when apparent motion was elicited using only bright or only dark components (Shechter and Hochstein, 1990). In this and other studies (Anstis, 1970; Anstis and Mather, 1985), motion perception was shown to deteriorate when a stimulus containing a dark and a bright element was used to generate apparent motion. This was interpreted as an indication that brightness polarity information is available to the motion system. We have confirmed these findings by determining threshold delays for successive onset of a dark and a bright line for a fixed separation of $3^{\prime}$ (diamonds in Fig. 2). At this separation, similar threshold delays are found for a pair of lines with equal contrast, either dark or bright. If our hypothesis is wrong, the signals of 
both pathways should have equal access to the neuronal substrate generating motion signals. Thus, we would expect similar thresholds (i.e., between 4 and $9 \mathrm{msec}$ ) as those for lines with equal contrast at a separation of $3^{\prime}$. If our hypothesis is correct, the thresholds measured under this condition should be much higher than for a pair of lines with equal contrast, implying that the perception of temporal order is achieved only at higher stages of the visual system. The latter expectation is substantiated by our results: in all subjects, temporal order was perceived correctly only when delays were at least four times as long as those required when two bright lines were presented in succession and at least twice as long as the delays required when two dark lines were presented in succession. These results independently confirm our findings and indicate that the first hypothesis outlined above is more likely to be true than the second.

Earlier results supporting the operation of two distinct physiological systems in the early stage of motion perception come from a study on the role of ON- and OFF-pathways in awake behaving monkeys. In these animals, $\mathrm{ON}$-center retinal ganglion cells were reversibly blocked by injecting a pharmacological agent (aminophosphonobutyrate) into the vitreous humor of the eye (Schiller et al., 1986). Detection of light increment but not of light decrement was severely impaired in these animals. The response to motion of a random dot pattern was only "mildly impaired," and an increase in the latency of saccadic responses to a moving target was found. These results are consistent with the conclusion drawn from our experiments: since the ON-center ganglion cells were inactivated, the neural system subserving ON-motion was blocked in these animals; however, the system subserving OFF-motion was still functioning, and therefore the response to motion of the random dot pattern was only mildly impaired. In our experiments, the thresholds observed for OFFmotion with a line separation larger than $3-4^{\prime}$ are significantly higher than the thresholds for ON-motion. Thus, the "mild" impairment as well as the increase in the saccadic latency found in the animals whose ON-center cells were blocked are in good agreement with our results. This result also indicates that the neural substrate responsible for ON-motion dominates in situations with moving random dots and supports the suggestion that ON- and OFF-center ganglion cells project to two physiologically distinct neural substrates computing motion.

\section{References}

Anstis SM (1970) Phi movement as a subtraction process. Vision Res 10:1411-1430.

Anstis SM, Mather G (1985) Effects of luminance and contrast on direction of ambiguous apparent motion. Perception 14:167-179.

Biederman-Thorson M, Thorson J, Lange GD (1971) Apparent movement due to closely spaced sequentially flashed dots in human peripheral field of vision. Vision Res 11:889-903.

Bolz J, Rosner G, Wässle H (1982) Response latency of brisk-sustained (X) and brisk-transient $(\mathrm{Y})$ cells in the cat retina. J Physiol (Lond) 328:171-190.

Exner S (1875) Experimentelle Untersuchungen der einfachsten psychischen Processe. Pfluegers Arch Gesamte Physiol Menschen Tiere 11:403-432.

Famiglietti EV Jr, Kolb II (1976) Structural basis for ON- and OFFcenter responses in retinal ganglion cells. Science 194:193-195.

Hubel DH, Livingstone MS (1990) Color and contrast sensitivity in the lateral geniculate body and primary visual cortex of the macaque monkey. J Neurosci 10:2223-2237.

Hubel DH, Wiesel TN (1968) Receptive fields and functional architecture of monkey striate cortex. J Physiol (Lond) 195:215-243.

Kremers J, Lee BB, Pokorny J, Smith VC (1991) The response of macaque retinal ganglion cells to complex temporal waveforms. In: From pigment to perception (Valberg A, Lee BB, eds), pp 173-176. New York: Plenum.

McKee SM (1981) A local mechanism for differential velocity detection. Vision Res 21:491-500.

Michelson A (1927) Studies in optics. Chicago: The University of Chicago Press.

Rodieck RW, Binmoeller KF, Dineen J (1985) Parasol and midget ganglion cells of the human retina. J Comp Neurol 233:115-132.

Schiller PH (1982) Central connections of the retinal ON and OFF pathways. Nature 297:580-583.

Schiller PH, Logothetis NK (1990) The color-opponent and broadband channels of the primate visual system. Trends Neurosci 13:392398.

Schiller PH, Sandell JH, Maunsell JHR (1986) Function of the ON and OFF channels of the visual system. Nature 332:824-825.

Shapley R (1990) Visual sensitivity and parallel retinocortical channels. Annu Rev Psychol 41:635-658.

Shechter S, Hochstein S (1990) ON- and OFF-pathway contributions to apparent motion perception. Vision Res 30:1189-1204.

Westheimer G (1983) Temporal order detection for foveal and peripheral visual stimuli. Vision Res 23:759-763.

Westheimer G, McKee S (1977) Perception of temporal order in adjacent visual stimuli. Vision Res 17:887-892.

Wiesel TN, Hubel DH (1966) Spatial and chromatic interactions in the lateral geniculate body of the rhesus monkey. J Neurophysiol 29: 1115-1156. 